\title{
The Effect of High Temperature Coolant and Lubricant on Cutting Process
}

\author{
Y. Zhang ${ }^{1 a}$, B.M.Li ${ }^{1}$, L.Han ${ }^{1}$, Q.Li ${ }^{1}$, L.P.Dong ${ }^{1}$ and Y.Q.Ma ${ }^{2}$ \\ ${ }^{1}$ School of Mechanical Engineering, Shenyang University of Technology, China \\ ${ }^{2}$ Shenyang YIXIN Science and Technology Development Company Ltd., China \\ azhy@sut.edu.cn, sutzhy@qq.com
}

Keywords: green cutting, coolant, lubricant, chip formation

\begin{abstract}
Green cutting is ecologically desirable and have been a tendency in the industry field. High temperature coolant and lubricant can be introduced in metal cutting due to its pollution-free, generating easily and unneeded disposal. This study attempts to understand the effect of high temperature coolant and lubricant on chip formation. In the comparison experiments to dry and wet cutting, high temperature coolant and lubricant flow from a developed generator is applied into cutting zone directly. When K20 tools are used to turn titanium alloy Ti-6Al-4V, Ni-based super alloy GH3030 and stainless steel 1Cr18Ni9Ti in orthogonal cutting, through quick-stop tests, the photos of polished chip sections microstructure were obtained. And the results suggest that the application of high temperature coolant and lubricant produces the least BUE, tool-chip contact length but the largest deformation coefficient and shear angle.
\end{abstract}

\section{Introduction}

Cutting fluids and additives are employed to decrease cutting forces and cutting temperature, prevent the formation of built-up edge, generally prolong tool life, enhance surface finish and remove swarf from the cutting area in metal cutting ${ }^{[1,2]}$. However, some of them, especially extreme pressure additives, contain $\mathrm{Cl}, \mathrm{S}$ or $\mathrm{P}$ compounds which endanger to environment and people health. Therefore, nowadays conventional cutting fluids and additives have not been seen as a solution, but a heavy burden of the environment. In the 21st century, it is a global tendency to developing green cutting technology concerned environment protection.

Water vapor, in 1990s, was applied as environment-friendly coolant and lubricant in metal cutting by Podgorkv V.V. and Godlevski V.A. ${ }^{[3]}$. It is reported that as the water vapor application carbide tool life was 1-1.5 times in turning and 1-3 times in milling longer than that in dry cutting for medium carbon and stainless steel. Our further research indicated that by using water vapor the cutting forces reduced 30\%-40\%, 10\%-15\% compared with dry and wet cutting in turning of medium carbon steel ${ }^{[4]}$. And the influences of nozzle diameter, jet flow parameters and cooling distance respectively on the cutting forces were presented in another study ${ }^{[5]}$.

This paper aims to investigate the effect of water vapor as coolant and lubricant on the chip formation of difficult-to cut materials, including titanium alloy, super alloy and stainless steel, compared with dry and wet cutting by quick-stop tests. The cutting parameters were conducted at constants. Microstructure photos of polished chip sections were obtained. And shear angle, tool-chip contact length and deformation coefficient have been examined for comparing the application of water vapor to dry and wet machining in orthogonal cutting.

\section{Experiments}

Exploding quick-stop device.The nature of flow in chip formation can be obtained by a quick-stop test with using an exploding quick-stop device. In metal cutting process, the cutting tool is accelerated by an impacting mass to a speed much greater than cutting speed in a time much less than that required for the chip to travel through its own thickness. And then the tool goes down in the high 
speed immediately, and the chip root left partially formed on the workpiece can be cut-away, sectioned and etched to show its form and microstructure. And the rake angle, $\gamma_{0}=0^{\circ}$, the clearance angle, $\alpha_{0}=14^{\circ}$ and the cutting edge inclination angle $\lambda_{s}=0^{\circ}$.

Cutting experiments. As shown in Fig.1, orthogonal cutting experiments were carried out using a center lathe CA6140, powered by a 7.5kw electric motor giving a speed range of 10 1400 rev/min and a feed range of $0.014 \sim 3.16 \mathrm{~mm} / \mathrm{rev}$. The cutting tools applied were YG8 (WC +8\%Co, K20 in ISO) uncoated parting tools and new tool was used for each experiment. The workpiece used were titanium alloy TC4 (Ti-6Al-4V), Ni-based super alloy GH3030, stainless steel 1Cr18Ni9Ti and comparison material medium carbon steel C45, as given in Tab.1 with cutting speed and the feed was $0.1 \mathrm{~mm} / \mathrm{rev}$. The lubricating conditions and parameters in the cutting experiments are given in the Tab.2.
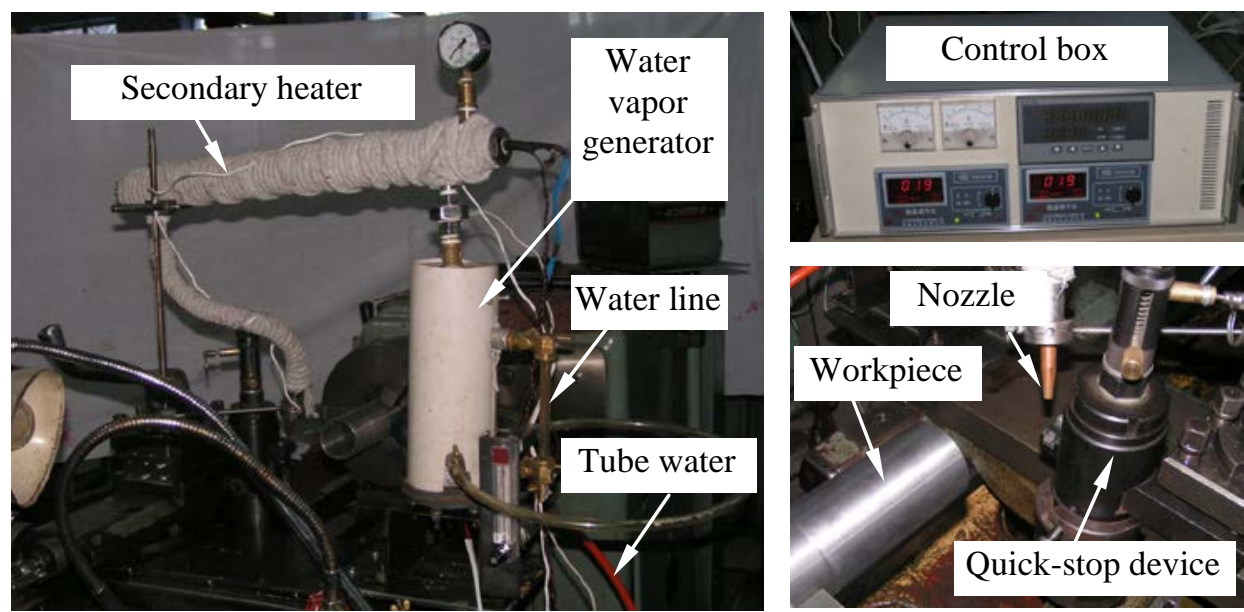

Fig.1 Experiments system

Tab.1 Workpiece materials and cutting speed

\begin{tabular}{|c|c|c|c|c|}
\hline Materials & $\begin{array}{c}\text { Main components } \\
\text { [wt \%] }\end{array}$ & $\begin{array}{c}\sigma_{\mathrm{b}} \\
{[\mathrm{MPa}]}\end{array}$ & $\begin{array}{c}\text { Geometry } \\
(\mathrm{D} \times \mathrm{B} \times \mathrm{L})[\mathrm{mm}]\end{array}$ & $\begin{array}{c}\text { Cutting speed } \\
{[\mathrm{m} / \mathrm{min}]}\end{array}$ \\
\hline TC4 & $\mathrm{Al} 6.10, \mathrm{~V} 4.22, \mathrm{O} 0.12, \mathrm{Fe} 0.06, \mathrm{C} 0.01$ & 980 & $60 \times 4 \times 200$ & 60 \\
\hline GH3030 & C0.35,Cr20.68,Fe0.61,Ti0.27,Mn0.42 & 750 & $52 \times 4 \times 200$ & 40 \\
\hline 1Cr18Ni9Ti & C0.045, Cr17.76,Ni9.62,Ti0.4,Mn1.14 & 580 & $60 \times 4 \times 200$ & 60 \\
\hline 45 & C0.45,Mn0.64,Si0.21,P,S $\leq 0.035$ & 640 & $\begin{array}{l}60 \times 4 \times 200 \\
52 \times 4 \times 200\end{array}$ & $\begin{array}{l}60 \\
40\end{array}$ \\
\hline
\end{tabular}

Tab.2 The lubricating conditions and parameters

\begin{tabular}{cccccc}
\hline \multirow{2}{*}{ Lubricating conditions } & \multicolumn{5}{c}{ Parameters } \\
\cline { 2 - 6 } & $\begin{array}{c}\text { Temperature } \\
{ }^{\circ} \mathrm{C}\end{array}$ & $\begin{array}{c}\text { Flux } \\
\text { L/min }\end{array}$ & $\begin{array}{c}\text { Pressure } \\
\text { MPa }\end{array}$ & $\begin{array}{c}\text { Cooling distance } \\
\text { mm }\end{array}$ & Concentration \\
\hline Dry cutting & & & & ---- & \\
Cutting fluid & 19 & 1 & 0.12 & 20 & $5 \%$ \\
Water vapor & 125 & 45 & 0.125 & 20 & --- \\
\hline
\end{tabular}

\section{Results and discussion}

Microstructure of chip section. The chip section microstructure can be observed by Zeiss metallographic microscope and those of using water vapor in cutting are shown in Fig.2. The direction of shear plane is represented by line $A B$, separating the deformed and undeformed regions. Tool-chip contact length, shear angle and deformation coefficient were examined to study the lubricating action of water vapor compared with dry and wet cutting, and each investigated subject was presented in different headings as given below. 


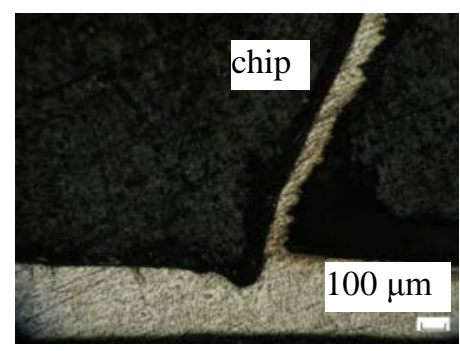

(a) TC4 60m/min

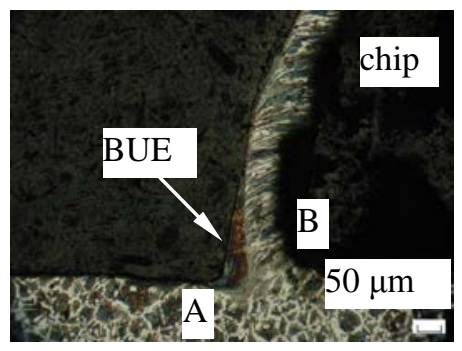

(d) C45 60m/min

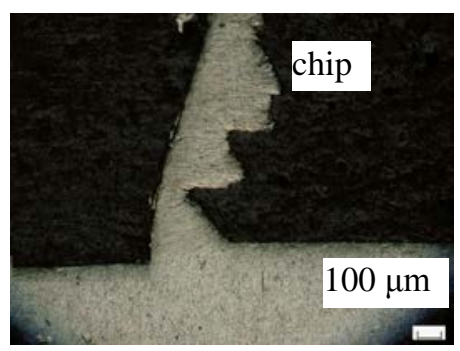

(b) $\mathrm{CH} 303040 \mathrm{~m} / \mathrm{min}$

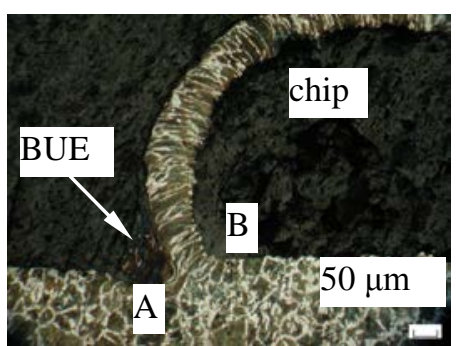

(e) $\mathrm{C} 4540 \mathrm{~m} / \mathrm{min}$

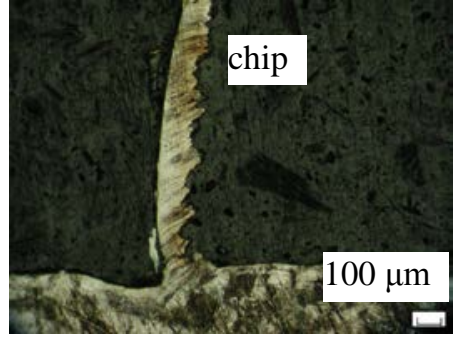

(c) $1 \mathrm{Cr} 18 \mathrm{Ni} 9 \mathrm{Ti} 60 \mathrm{~m} / \mathrm{min}$

Etched by

TC4: $\mathrm{HF}+\mathrm{HNO}_{3}+\mathrm{H}_{2} \mathrm{O}$

GH3030: $\mathrm{HCl}+\mathrm{HNO}_{3}+\mathrm{H}_{2} \mathrm{O}$

1Cr18Ni9Ti: $\mathrm{CuSO}_{4}+\mathrm{HCl}+\mathrm{H}_{2} \mathrm{O}$

C45: $\mathrm{HNO}_{3}+\mathrm{C}_{2} \mathrm{H}_{5} \mathrm{OH}+\mathrm{H}_{2} \mathrm{O}$

Fig.2 The chip section microstructures of water vapor as coolant and lubricant

Tool-chip contact length.The tool-chip contact length can be obtained from the photomicrograph and the results are shown in Fig.3. For all materials, the using of coolant and lubricant in the cutting zone gave a decreased tool-chip contact length. And the application of water vapor produced the shortest tool-chip contact length according to Fig.3. The short tool-chip contact length implied that the shear stress on the rake face was lower. Therefore, the lubricating action of water vapor is more effective than that of cutting fluid in cutting. It is the formation of BUE that presents the most tool-chip contact length for C45, compared with difficult-to-cut materials, and the effect of water vapor restraining the BUE is better than that of cutting fluid.

Shear angle.The shear angle, which is an important parameter in metal cutting, was measured directly and the results are shown in Fig.4. It is clear that the shear angle is affected by coolant and lubricant conditions, and the application of water vapor produced the highest shear angle for all experimental materials. The increasing shear angle will lower the friction coefficient on the rake face according to shear angle theory; and the friction force and the heat generating from friction on the tool rake face are all lower, in hence, the cutting forces and temperature would be all decreased, at last the tool life could be prolonged.

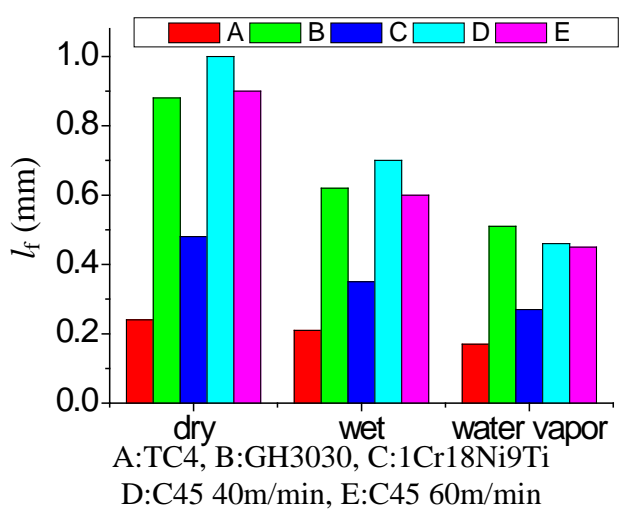

Fig.3 Tool-chip contact length

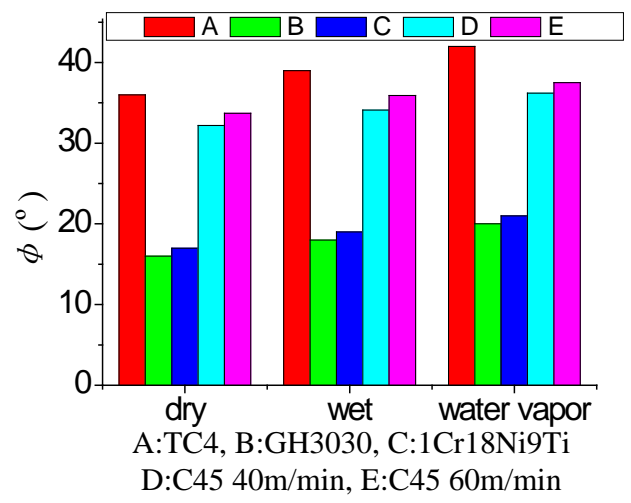

Fig.4 Shear angle

Deformation coefficient.Along with the increasing of shear angle, deformation coefficient has a reduce trend, as shown in Fig.5. It is noted that the titanium alloy takes the character of small deformation coefficient and the coolant and lubricant produces a tiny impact, but contrarily, because of the big deformation for super alloy and stainless steel with in cutting, the effect of coolant and lubricant is more obvious. For difficult-to-cut materials and general materials, the lubricating action of water vapor decreased the deformation coefficient. And the reducing deformation coefficient 
indicated that the resistance force acting on the tool rake face and the heat generating of cutting layer deformation are all lower, and then as a result, the cutting forces and cutting temperature reduce correspondingly.

\section{Conclusions}

The quick-stop experimental investigation of water vapor as coolant and lubricant, dry and wet cutting in the turning of titanium alloy TC4, super alloy Gh3030, stainless steel 1Cr18Ni9Ti and medium carbon steel C45 with YG8 tool was carried out. The aim of the study was to examine the effect of water vapor as coolant and lubricant on chip formation compared with dry and wet cutting. The study provided some results as follow:

(1) For difficult-to-cut materials, from the examining results, it is visible that the application of water vapor in cutting produces the shortest chip-tool contact length and the least deformation coefficient but the biggest shear angle, compared with dry and wet cutting.

(2) It is indicated that the lubricant action of water vapor is better than cutting fluid, and its well lubricant action in metal cutting comes from its effective osmosing into capillaries in cutting zone.

(3) The excellent osmosis and lubricant action of water vapor in difficult-to-cut materials cutting could decrease the cutting forces and cutting temperature, enhance the tool life.

(4) Water vapor is free of pollution, easily to be generated and no needed disposal and could be a potential solution of green cutting considering preventing the pollution of the environment instead of cutting fluids, according to the results of experiments.

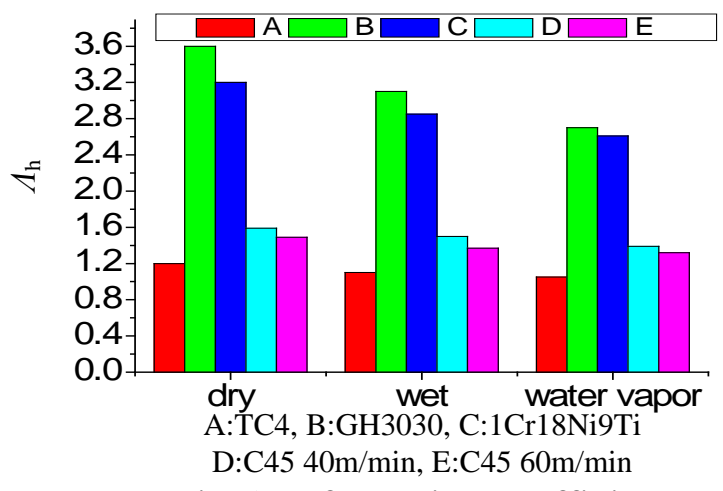

Fig.5 Deformation coefficient

\section{Acknowledgements}

This research reported in the paper is financially supported by Foundation of Liaoning Province Education Department (L2012042), Doctoral Research Foundations of Liaoning Province (20121058). These supports are greatly acknowledged.

\section{References}

[1] M.A.EI Baradie: Journal of Materials Processing Technology vol.56 (1996), p.786-p.797

[2] E.M Trent: Metal cutting (3rd Ed.) (Butterworth Heinemann, Oxford 1991)

[3] R.D. Han: New Technology of Machining in Modern Times (in Chinese) (China Publishing House of Electronics Industry, Beijing 2003)

[4] J.Y. Liu, R.D. Han and Y.F. Sun: International Journal of Machine Tools and Manufacture vol.45 (2005), p.687-p.694

[5] R.D. Han, J.Y. Liu , Y. Zhang and L. Zhang: Key Engineering Materials vol.315-316(2006), p.45-p.50 